\title{
Developmental cycle of chelonian haemogregarines in leeches with extra-intestinal multiple sporozoite oocysts and a note on the blood stages in the chelonian hosts
}

\author{
Ilan Paperna \\ Department of Animal Sciences, Faculty of Agriculture of the Hebrew University of Jerusalem, Rehovot, 76-100, Israel
}

\begin{abstract}
Gametocytes and oocysts of chelonian haemogregarines: Haemogregarina pelusiensi and Haemogregarina sp. are described from natural leech hosts: Placobdella multistrigata and P. costata. The chelonian hosts were Pelusios sinuatus from South Africa and Mauremys caspica from Israel, respectively. Oocyst stages in leeches migrate from the intestine into the surrounding connective tissue and form over 40 naked sporozoites. Blood stages from both chelonian hosts are similar in structure and size despite their hosts representing very distinct chelonian taxa.
\end{abstract}

\section{INTRODUCTION}

Accounts have been given on the development in leech of Haemogregarina stepanowi by Reichenow (1910) (type species of the genus Haemogregarina) of $H$. nicoriae by Robertson (1910) and of $H$. balli by Paterson \& Desser (1976). These reports describe gametocytes attached to the leech's intestinal epithelial surface and developing via syzygy into small oocysts. The latter divide into 8 naked sporozoites while still attached to the intestine. All 3 of these haemogregarines are parasites of chelonians (in Europa, Sri-Lanka and USA respectively).

The present communication describes gamogonous development of haemogregarines from the chelonians Pelusios sinuatus from South Africa and Mauremys caspica from Israel, in leeches which yield extraintestinal oocysts with numeous sporozoites. A brief account on the blood stages in the chelonian hosts is provided.

\section{MATERIALS AND METHODS}

Leeches fixed in neutral buffered formalin (10\%) were processed for methyl-methacrylate/glass knife histology by the Lulham (1979) method. Sections, 3 to 4 um thick, were stained by Meyer's Haemalum-Eosin-
Phloxine (H-E-P). Blood smears from chelonians were taken from the clipped tip of the tail. Air dried smears were fixed with absolute methyl alcohol and stained 15 min in $1 / 7$ diluted Giemsa stock with $\mathrm{pH} 6.8$ phosphate buffer.

\section{RESULTS}

Developmental stages were demonstrated in: (a) the leech Placobdella multistrigata, found attached and feeding on naturally infected Pelusios sinuatus obtained from the vicinity of Pietersburg, northern Transvaal, South Africa; (b) the same species of leeches and chelonian from the same locality, maintained for $1 \mathrm{yr}$ in a laboratory aquarium; (c) laboratory bred Placobdella costata examined 12 to 15 d after taking their 2nd or 3rd blood meal from naturally infected Mauraemys caspica from Lake Kinnereth, Israel.

In both Placobdella multistrigata and $P$. costata, the gastric ceaca contents included released trophozoites and encapsulated, rarely released gametocytes, but never further developmental stages. In both leeches, macrogametocytes associated with microgametocytes and zygotes were found attached to the surface of the epithelium of the intestine (Figs. 1 to 6). Such macrogametocytes and zygotes measured 7 to $8 \times 6$ to $7 \mu \mathrm{m}$ 

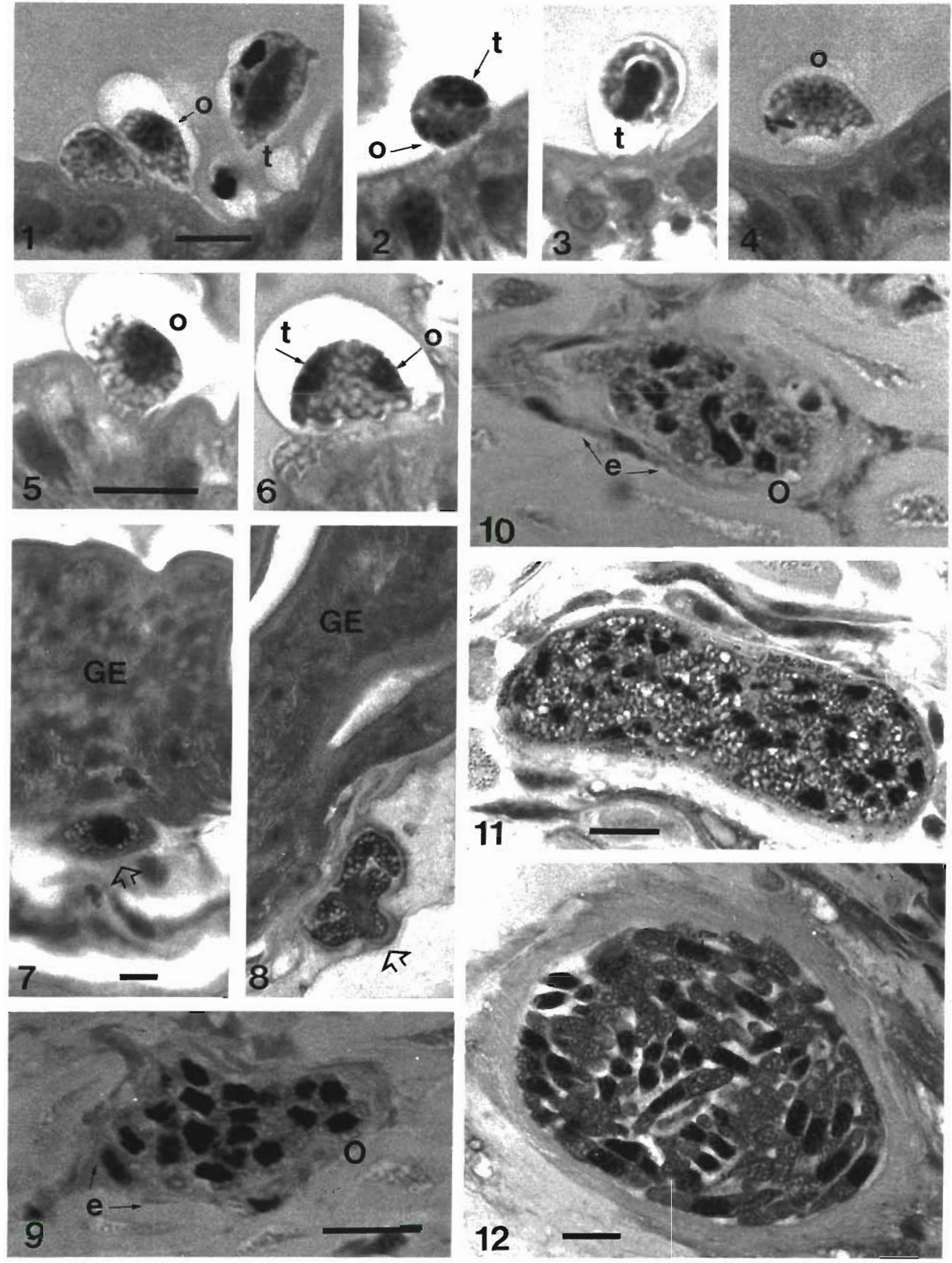

Figs. 1 to 12. Placobdella spp. Histological sections; Meyer's Haemalum-Eosin-Phloxin; scale bar = $10 \mu \mathrm{m}$. Figs. 1 to 6 . Macrogametocytes $(0)$ attached to brush border of leech's intestinal epithelium, some in association with microgametocytes (t); Figs. 1 to 4 and 6. P. costata; Fig. 5. P. multistrigata. Fig. 7. P. multistrigata. Zygote/young oocyst (arrow) located beneath intestinal epithelium (GE). Fig. 8. P. multistrigata. Dividing oocyst located beneath intestinal epithelium (GE); 5 cale bar as in Fig. 7. Figs. 9, 10. P. costata. Two section plans of oocyst (O) with $>16$ nuclei in connective tissue, e: host connective envelope. Fig. 11 . $P$. $\overline{m u}$ itistrigata. Oocyst containing $>30$ nuclei or already forming sporozoites. Fig. 12. P. multistrigata. Mature oocyst with $>40$ 

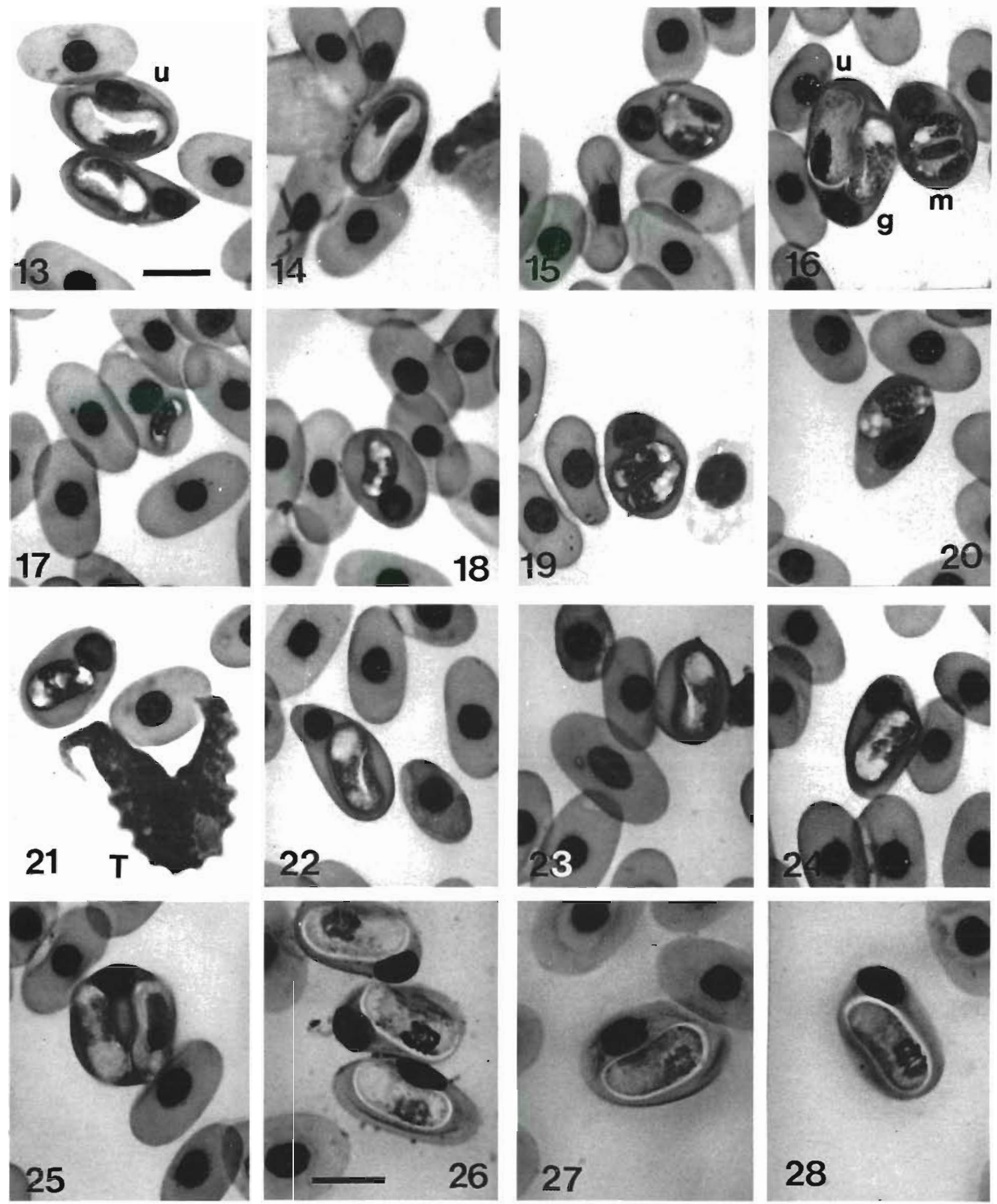

Figs. 13 to 28. Intraerythrocytic stages; Giemsa stain, scale bar $=10 \mu \mathrm{m}$. Figs. 13 to 25: Pelusios sinuatus. Figs. 13, 14. Bent trophozoites $(u)$. Fig. 15. Dividing meront. Fig. 16. Dividing meront $(\mathrm{m})$ and double infection with trophozoite (u) and a gametocyte

(g); Figs. 17 to 21. Developing gametocytes. Fig. 21. A trypanosome (T). Figs. 22 to 24. Mature gametocytes. Fig. 25. Double gametocyte infection. Figs. 26 to 28. Mauremys caspica. Gametocytes from blood 
in size, in $P$. multistrigata and 8 to $14 \times 5$ to $9 \mu \mathrm{m}$ im $P$. costata. In $P$. multistrigata, zygotes or young undivided oocysts 13 to $29 \times 6$ to $24 \mathrm{~km}$ in size (Fig. 7) and dividing oocysts (Fig. 8), loaded with translucent granules, were found in the connective tissue beneath the intestinal epithelium layer. In $P$. costata only 1 oocyst was found, located in the connective tissue, $24 \times 11$ to $13 \mu \mathrm{m}$ in size, with 15 nuclei (Figs. 9 and 10 ). Advanced stage oocysts with more than 40 nuclei, $66 \times 22 \mu \mathrm{m}$ in size (Fig, 11) and an oocyst containing over 55 sporozoites, $61 \times 44 \mu \mathrm{m}$ in size (Fig. 12) were found in the connective tissue of $P$. multistrigata. Oocysts in the leeches' connective tissue were either in close proximity to the intestine, or in a more distant location. Sporozoites within the oocyst, with a prominent nucieus and numeduus refractile granules, measured 19 to $26 \times 3$ to $7 \mu \mathrm{m}$ (Fig. 12). Free sporozoites were not seen in the leeches' tissues. Erythrocytic stages in the gastric ceaca persisted beyond the peroid during which oocysts sporulated.

\section{Note on the haemogregarine intraerythrocytic stages in the peripheral blood of the chelonian hosts}

Pelusios sinuatus, collected near Pietersburg, North Transvaal, South Africa (3 naturally infected specimens), contained tightly bent trophozoites ('serpentine schizonts' of Paterson \& Desser 1976) measuring 28 to $33 \times 4$ to $6 \mu \mathrm{m}$ and were enclosed within a 14 to $17 \times 4$ to $6 \mu \mathrm{m}$ capsule (Figs. 13, 14 and 16). Dividing meronts were rare, even in non-peripheral blood (lungs); meronts with 2 or 3 nuclei measured 15 to $17 \times 12$ to $13 \mu \mathrm{m}$ (Figs. 15 and 16).

Young gametocytes ranged in size from $7 \times 3$ to $15 \times 8 \mu \mathrm{m}$, were not encapsulated and lacked tapering ends (Figs. 17 to 21). Gametocytes, apparently mature, were 13 to $24 \times 5$ to $9 \mu \mathrm{m}$, stout, with 1 end tapering and tightly bent and enclosed in a bean-shaped, 9 to $17 \times 5$ to $9 \mu \mathrm{m}$ translucent, hard capsule. The nucleus was dendritic and translucent vacuoles were observed aggregated in the stout end of the gametocyte (Figs. 22 to 25). Differentiation into micro- and macrogametocytes was not possible. Infected erythrocytes became rounder and often were also distorted. A few erythrocytes contained 2, either young or mature, parasites (Figs. 16, 19 and 25).

Pelusios sinuatus were concurently infected by a trypanosome (Fig. 21) which appeared also in the foregut of $P$. multistrigata.

Mauremys caspica collected from Lake Kinnereth riverine systems, Israel ( 6 naturally infected specimens) contained tightly bent trophozoites 30 to $\times 4$ to $6 \mu \mathrm{m}$ in size, and enclosed in 15 to $16 \times 4$ to $7 \mu \mathrm{m}$ capsules. Dividing meronts were seen only in blood smears from the lung. Gametocytes, 21 to $28 \times 4$ to $9 \mu \mathrm{m}$, with a dendritic nucleus, were stout, at one end tapering and tightly bent. Gametocytes were enclosed in hard, translucent 15 to $19 \times 5$ to $9 \mu \mathrm{m}$ capsules (Figs. 26 to 28). Differentiation as micro- or macrogametocytes was not possible. Young gametocytes ( $<13 \times 4 \mathrm{um}$ in size) were rarely found, and infected erythrocytes were either normal or slightly altered in shape.

\section{DISCUSSION}

Haemogregarines from Pelusios sinuatus are apparently conspecific with Haemogregarina pelusiensi, reported by Pienaar (1962) in the same host species from Rio do Save, Mozambique.

Haemogregarines from Mauremys caspica from Israel are of the same populations, and therefore identical in dimensions to Haemogregarina sp. reported by Desser \& Yekutiel (1987) from the same host and localities. Although Pelusios sinuatus and $M$. caspica belong to different chelonian suborders (Pleurodira and Cryptodira, respectively), both the erythrocytic and the leech stages from the Israelian and the South African hosts were similar in shape and were overlapping in dimensions.

Khan (1978) and Lainson (1984) described piscine haemogregarines producing oocysts with numerous sporozoites in the leech host. Lainson (1984) suggested that haemogregarines producing more than 8 naked sporozoites cannot, by definition, be included in the genus Haemogregarina. He therefore proposed a new genus Cyrilla for these haemogregarines. The haemogregarines described herein diverge further from all previously described haemogregarines by virtue of the extra-intestinal location of the oocysts in the leech. In C. gomesi described by Lainson (1984), as in species of chelonian Haemogregarina (Reichenow 1910, Robertson 1910, Patterson \& Desser 1976) the oocyst develops and releases sporozoites while remaining attached to the brush border of the gut epithelium. Oocysts of $C$. uncinata (Khan 1978, Lainson 1984) sporulate while within the gut epithelium, sporozoites, nevertheless, are released into the intestinal lumen. Both authors consider transmission to occur when the leech feeds on the blood of the host fish.

Observation of sporulated oocysts, located in the extra-intestinal tissue, implies that unless sporozoites released from the oocysts are able to find another route to the leech mouth parts, the options for transmission are, in this case, limited to ingestion of the leech.

It seems that the sporogony of more haemogregarines should be studied before we can offer a rational system for generic division, which takes account of the options for transmission among haemogregarines pro- 
vided through the versatility in sporulation sites and sporozoite yields in the leech host.

Acknowledgements. I wish to thank Professor J. G. VanAs of the Department of Zoology and Entomology, Orange Free State University, Bloemfontem, my host in South Africa, for providing laboratory facilities and animals for study, Dr J. H Oozthuizen of the department of Zoology, University of Pretoria for the identification of the leeches. I wish also to thank Dr P. J. Irwin, Graduate School of Tropical Veterinary Science, James Cook University, Queensland, Australia for critical reading of the manuscript.

\section{LITERATURE CITED}

Desser, S. S., Yekutiel, D. (1986/87). Blood parasites of amphibians and reptiles in Israel. Israel J. Zool. 34: 77-90

Responsible Subject Editor: Professor P. Zwart, Utrecht, The Netherlands
Khan, R. A. (1978). A new haemogregarine from marine tishes. J. Parasit. $64: 35-44$

Lainson, R. (1984). On Cyrilla gomesi (Neiva \& Pinto, 1926) gen. nov. (Haemogregarinidae) and Trypanosoma bourouli Neiva \& Pinto, in the fish Synbranchus marmoratus simultaneous transmission by the leech Haementeria lutzi. J. Protozool. 31: 150-158

Lulham, C. N. (1979). Glycol. methacrylate embedding for light microscopy. J. Histotechnol. 2: 68-71

Paterson, W. B., Desser, S. S. (1976). Observations on Haemogregarina balli sp. nov. from the common snapping turtle Chelydra serpentina. J. Protozool. 23: 294-301

Pienaar, U. de V (1962). Haematology of some South African reptiles. Witwatersrand Univ. Press, Johannesburg

Reichenow, E. (1910). Haemogregarina stepanowi. Die Entwicklungsgeschichte einer Haemogregarine. Arch. Protistenk. 20: 251-350

Robertson, M. (1910). Studies on Ceylon haematozoa. No. II Notes on the live cycle of Haemogregarina nicoriae Cast. and Willey, 1904. Q. J. Microsc. Sci. 55: 741-762

Revised version accepted: June 14, 1989 